\title{
Los factores de riesgo modificables en la enfermedad cardiovascu- lar son similares en 52 países
}

\section{Objetivo}

Determinar la fuerza de asociación entre varios factores de riesgo conocidos y el primer infarto agudo de miocardio (IAM) en diferentes regiones geográficas, etnias, sexo y edad.

Diseño

Estudio caso-control internacional.

Lugar

262 centros de 52 países (incluida Argentina) de todos los continentes habitados.

\section{Participantes}

Incluyeron 15.152 (pacientes de cualquier edad que ingresaron en unidades de cuidados intensivos cursando su primer IAM, dentro de las 24 hs de iniciados los síntomas) y 14.820 controles que se reclutaron del ámbito hospitalario y de la comunidad, sin antecedentes coronarios, que fueron apareados* por sexo y edad.

Evaluación de los factores de riesgo y medición de resultados Se administraron cuestionarios donde se obtuvo información acerca de factores demográficos y socioeconómicos, tabaquismo (TBQ), tiempo libre, actividad física, alimentación, historia personal y familiar de enfermedad cardiovascular, hipertensión arterial (HTA), diabetes (DBT), depresión, percepción de estrés. Se realizó un examen físico y se tomaron muestras de sangre en las primeras $24 \mathrm{hs}$. del ingreso. Como medida de riesgo se utilizó el Odds ratio $(\mathrm{OR})$ ajustado* y el riesgo atribuible poblacional (sigla en inglés PAR)* con un IC $99 \%$ para dichos factores de riesgo (FR) y sus combinaciones.

\section{Resultados principales}

La mediana de edad de aparición del primer IAM fue de 9 años menor en hombres que en mujeres en todas las regiones del mundo. Todos los FR se asociaron significativamente a la aparición de IAM, excepto el consumo de alcohol, que tuvo una asociación más débil y con tendencia protectora. Entre ellos, el TBQ (OR 2,87; IC95\% 2,58-3,19, PAR $37 \%$ ) y la dislipemia (OR 3,25; IC 2,813,76 , PAR $49,5 \%$ ) fueron los factores asociados con mayor fuerza al primer IAM, seguidos por los factores psicosociales, la DBT y la HTA (ver tabla 1). Se halló una importante relación entre el número de cigarrillos consumidos y el riesgo de IAM. En individuos que fumaban más de 40 cigarrillos/día el OR fue de 9,16 (IC 6,18-13,58) y disminuyó en forma significativa a medida que el consumo de tabaco era menor. También se observó una relación entre mayor dislipemia y riesgo de IAM. La circunferencia abdominal evaluada a través de la relación cintura-cadera fue mejor predictor de riesgo que el índice de masa corporal, y en los países con altos y medianos ingresos la obesidad abdominal se asoció con un PAR mayor que el del tabaquismo. El consumo diario de frutas y verduras, la actividad física y el consumo de alcohol (3 o más veces en la semana) fueron protectores frente al IAM. Juntos, el TBQ, HTA y DBT incrementan OR de IAM a 13,01 (IC 10,69-15,83), y explican el $53 \%$ del PAR. La combinación de dislipemia y TBQ explican un PAR de $66,8 \%$ y los 9 FR independientes confieren un OR de 129,2 (IC 90,24 -184,99), representando un PAR de 90,4\% (IC 88,1$92,4)$, ligeramente mayor para las mujeres (PAR 94\%). No se observaron diferencias por edad ni variaciones étnicas.

Tabla 1: Riesgo de IAM asociado con los distintos factores de riesgo y riesgo atribuible poblacional.

\begin{tabular}{l|c|c}
\multicolumn{1}{c|}{ Factor de riesgo } & Odds ratio (IC99\%) ajustado* & PAR (IC99\%) \\
\hline Tbq & $2,87(2,58-3,19)$ & - \\
\hline Tbq y ex tbq & $2,04(1,86-2,25)$ & $35,7 \%(32,5-39,1)$ \\
\hline Dbt & $2,37(2,07-2,71)$ & $9,9 \%(8,5-11,5)$ \\
\hline Hta & $1,91(1,74-2,10)$ & $17,9 \%(15,7-20,4)$ \\
\hline Obesidad abdominal (tercilos 2 vs 1) & $1,12(1,01-1,25)$ & - \\
\hline Obesidad abdominal (tercilos 3 vs 1) & $1,62(1,45-1,80)$ & $20,1 \%(15,3-23,0)$ \\
\hline Factores psicosociales & $2,67(2,21-3,22)$ & $32,5 \%(25,1-40,8)$ \\
\hline Consumo diario de frutas y verduras & $0,70(0,62-0,79)$ & $13,7 \%(9,9-18,6)$ \\
\hline Ejercicio regular & $0,86(0,76-0,97)$ & $12,2 \%(5,5-25,1)$ \\
\hline Consumo regular de alcohol & $0,91(0,82-1,02)$ & $6,7 \%(2,0-20,2)$ \\
\hline Dislipemia & $3,25(2,81-3,76)$ & $49,2 \%(43,8-54,5)$ \\
\hline Todos los factores de riesgo comb. & $129,2,(90,24-184,99)$ & $90,4 \%(88,1-92,4)$ \\
\hline
\end{tabular}

* Para todos los otros factores de riesgo, ver glosario

\section{Conclusiones}

La dislipemia, el tabaquismo, la hipertensión arterial, la diabetes, la obesidad abdominal, el estrés psicosocial, el bajo consumo de frutas, vegetales y alcohol y la falta de actividad física explican más del $90 \%$ de riesgo atribuible para un primer infarto, sin distinción de sexo, para distintas regiones del mundo y grupos étnicos. Aunque las prioridades pueden ser dispares en las distintas regiones. Los esfuerzos en la prevención de estos factores pueden basarse en los mismos principios en todo el mundo y prevenir así la mayoría de los IAM.

\section{Comentario}

La enfermedad cardiovascular (ECV) es una de las principales causas de morbi-mortalidad a nivel mundial. Si bien las muertes se redujeron en la última década en los países desarrollados, no sucedió lo mismo en los de bajos y medianos ingresos, en donde se detectó un incremento significativo de las mismas ${ }^{1,2}$. Los autores de este trabajo quisieron investigar si, además de ratificar la asociación entre los factores de riesgo cardiovasculares (FRC) y el IAM, ésta se mantenía inalterada a nivel mundial, en diferentes países con diferentes costumbres y etnias. Otro punto a evaluar fue el impacto absoluto que cada FRC tiene en relación al riesgo global de infartarse de la población. Este es el primer estudio internacional de gran envergadura que evaluó nueve factores de riesgo fácilmente cuantificables en un importante número de pacientes alrededor de todo el mundo, intentando averiguar qué relevancia tendrían los factores psicosociales en la incidencia de enfermedad coronaria.

El estudio encontró que los FRC clásicos medidos (tabaquismo, HTA, dislipemia y diabetes) y otros como la obesidad central (índice cintura/cadera), factores nutricionales (ingesta reducida de alcohol, frutas y verduras), escasa actividad física y niveles elevados de estrés, ansiedad y depresión se asocian fuertemente a IAM, independientemente de la nacionalidad, etnia o sexo. Estos factores actuando juntos son responsables de casi todos (más del
$90 \%$ ) los infartos mundiales.

Son interesantes algunas particularidades, como la mayor utilidad del índice cintura/cadera que la medida del índice de masa corporal para valorar riesgo cardiovascular, hecho que nos hace pensar en la asociación de éste con el síndrome metabólico, y la posibilidad de considerar al estrés, la ansiedad y la depresión como factores de riesgo cardiovascular independientes, ya que la probabilidad de tener un IAM es mayor al doble en presencia de estos factores, que por sí solos, explican el 30\% del riesgo atribuible*. Lo alentador de este trabajo es que parece que con cambios en el estilo de vida y buen control de los FRC clásicos, se podrían obtener resultados con gran impacto epidemiológico, reduciendo la incidencia y la mortalidad del infarto agudo de miocardio, en todas las poblaciones del mundo. Las dietas ricas en frutas y verduras, por ejemplo, asociadas con la práctica de actividad física regular, disminuyen la probabilidad de padecer un IAM en un $40 \%$, y si además, se logra la supresión del consumo de tabaco, en forma conjunta estos factores reducen la probabilidad de un IAM en casi el $80 \%$. Ahora bien, ¿Podríamos los médicos en nuestra práctica diaria intervenir sobre los factores estresores del trabajo y el hogar de nuestros pacientes? ¿Deberíamos hacer rastreo de depresión para reducir el riesgo cardiovascular? Puntos que pueden darse a discusión, pero no hay duda de que el interheart, más allá de las 


\section{El estrés psicosocial incrementa el riesgo de infarto de miocardio}

\section{Objetivo}

Investigar la relación entre los factores de riesgo psicosociales y el infarto agudo de miocardio (IAM).

Diseño

Estudio caso-control.

\section{Lugar}

52 países (incluida Argentina) de todos los continentes habitados.

\section{Pacientes}

Se incluyeron 11.119 casos con IAM dentro de las primeras $24 \mathrm{hs}$. del ingreso. Se incluyeron 13.648 controles sin antecedentes cardiológicos, reclutados en los mismos centros, entre los visitantes de los pacientes casos o parientes sin enfermedad cardiológica o pacientes atendidos en los centros por causas no cardiológicas.

\section{Evaluación de los factores de riesgo}

Los participantes respondieron cuestionarios de cuatro preguntas simples acerca de estrés en el hogar y en el trabajo, estrés financiero y eventos mayores de la vida en el último año (como separación, muerte, enfermedad mayor, violencia, conflicto intrafamiliar, etc), nivel de control en el manejo de situaciones estresantes de la vida o depresión. Idearon un cuestionario de cuatro preguntas simples para evaluar estrés como por ejemplo "experimenté estrés a veces, frecuentemente o permanentemente en el trabajo o en el hogar". Se preguntó además sobre factores demográficos (país de orígen, idioma nativo), nivel socioeconómico (educación, ocupación, ingresos), y otros factores de riesgo conocidos (ver resumen de hoja previa).

\section{Medición de resultados principales}

Evaluaron la relación independiente del estrés y la aparición del primer IAM, teniendo en cuanta el resto de los factores de riesgo tradicionales. Como medida de riesgo se utilizó el Odds ratio (OR) ajustado* y el riesgo atribuible poblacional (sigla en inglés PAR)* con un IC $99 \%$ para dichos factores de riesgo.

\section{Resultados principales}

Los casos reportaron una mayor prevalencia de todos los factores estresores tenidos en cuenta en el estudio que los controles. Ver tabla 1. EI PAR de las variables osciló entre el 8 y $16 \%$, y colectivamente se observó un PAR del $33 \%$.

Tabla 1. Asociación entre IAM y factores psicosociales

\begin{tabular}{l|c|c}
\multicolumn{1}{c|}{ Factor psicosocial } & OR (IC 99\%) ajustado* & PAR (IC99\%) \\
$\begin{array}{l}\text { Frecuentes períodos de estrés en el } \\
\text { trabajo }\end{array}$ & $1,38-(1,19-1,61)$ & - \\
\hline Estrés permanente en el trabajo & $2,14-(1,73-2,64)$ & $9 \%(1-18)$ \\
\hline $\begin{array}{l}\text { Frecuentes períodos de estrés en el } \\
\text { hogar }\end{array}$ & $1,52-(1,34-1,72)$ & - \\
\hline Estrés permanente en el hogar & $2,12-(1,68-2,65)$ & $8 \%(4-12)$ \\
\hline Estrés financiero severo & $1,33-(1,19-1,48)$ & $11 \%(7-14)$ \\
\hline $\begin{array}{l}\text { Situaciones adversas de la vida en } \\
\text { el año previo (2 o +) }\end{array}$ & $1,48-(1,33-1,64)$ & $10 \%(8-13)$ \\
\hline Depresión & $1,55-(1,42-1,69)$ & $9 \% 7-10)$ \\
\hline
\end{tabular}

* Para otros factores de riesgo, para definición de ajuste ver glosario.

\section{Conclusión}

Los estresores psicosociales se asociaron de manera independiente y significativa con un incremento en el riesgo de infarto agudo de miocardio.

Si esta asociación es verdaderamente causal, la importancia de factores psicosociales sería mucho más importante de lo habitualmente reconocido y contribuiría a una proporción sustancial de los IAM en el mundo. limitaciones metodológicas para cuantificar estos factores psicosociales, es el primer estudio de gran magnitud que los evalúa en el contexto de los FR tradicionales.

Los resultados del INTERHEART son interesantes ya que son aplicables a todas las regiones geográficas, incluyendo la Argentina, que colaboró en el estudio. En nuestro país tenemos adecuada información sobre prevalencia de los FRC como para encarar serias medidas de prevención, aún teniendo en cuenta que coexisten muy distintas rea-lidades dentro de nuestro suelo'. Hay amplios grupos poblacionales que no tienen acceso a la alta tecnología para la prevención y tratamiento del infarto agudo de miocardio. $Y$ he aquí la máxima utilidad del INTERHEART: nos dice que con estrategias serias dirigidas a promover cambios en el estilo de vida, se podrían obtener resultados con gran impacto epidemiológico, reduciendo la incidencia y la mortalidad del IAM.

\section{Conclusión de las comentadoras:}

Con estrategias poblacionales destinadas a combatir el tabaquismo, el sedentarismo y el estrés, y a estimular la ingesta de una dieta saludable, es posible reducir el riesgo poblacional de sufrir un IAM en forma considerable, independientemente del sexo y origen étnico de la población.

* ver glosario

Dras. María Eugenia Navarro, Noelia Capellato y Victoria Wurcel [Unidad de Medicina Familiar y Preventiva. Hospital Italiano de Buenos Aires.]

Navarro M, Capellato N, Wurcel V. Los factores de riesgo modificables en la enfermedad cardiovascular son similares en 52 países. Evid.actual.práct.ambul. 2005;14-15. Comentado de Effect of potentially modifiable risk factors associated with myocardial infarction in 52 countries (the INTERHEART study): case-control study. Yusuf S, Hawken S, Ounpuu S y col Lancet 2004; 364:937-52. PMID: 15364185. El estrés psicosocial incrementa el riesgo de IAM. Comentado de: Association of psychosocial risk factors with risk of actue myocardial infraction in 11119 cases and 13648 controls from 52 countries (the INTERHEART study):case-control study. Rosengren A, Hawken S,Ounpuu S .y col. Lancet 2004; 364:953 -962. PMID: 15364186

\section{Referencias}

1. Área de Investigación, Consejo de Epidemiología y Prevención Cardiovascular, Área del Interior y Fundación Cardiológica SAC. Estudio Redifa. Rev Argent Cardiol 2002; 70: 300-311 2. Zylbersztejn H. Comentario del INTERHEART. Web SAC. 2004. 